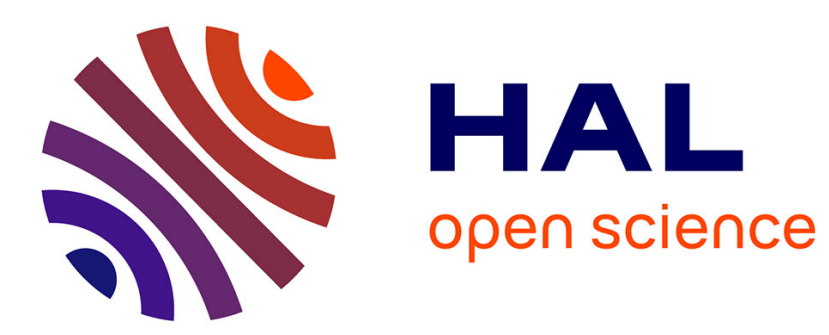

\title{
A GAIN SCHEDULED CONTROL LAW FOR FIN/RUDDER ROLL STABILISATION OF SHIPS
}

Hervé Tanguy, Guy Lebret

\section{To cite this version:}

Hervé Tanguy, Guy Lebret. A GAIN SCHEDULED CONTROL LAW FOR FIN/RUDDER ROLL STABILISATION OF SHIPS. conférence IFAC CAMS 2004 : Control Applications in Marine Systems, Jul 2004, Ancone, Italy. pp.107-112. hal-00369652

\section{HAL Id: hal-00369652 https://hal.science/hal-00369652}

Submitted on 20 Mar 2009

HAL is a multi-disciplinary open access archive for the deposit and dissemination of scientific research documents, whether they are published or not. The documents may come from teaching and research institutions in France or abroad, or from public or private research centers.
L'archive ouverte pluridisciplinaire HAL, est destinée au dépôt et à la diffusion de documents scientifiques de niveau recherche, publiés ou non, émanant des établissements d'enseignement et de recherche français ou étrangers, des laboratoires publics ou privés. 


\title{
A GAIN SCHEDULED CONTROL LAW FOR FIN/RUDDER ROLL STABILISATION OF SHIPS
}

\author{
Hervé Tanguy ${ }^{*, * *}$ Guy Lebret ${ }^{* *}$ \\ * SIREHNA: Nantes - France - www.sirehna.com \\ ${ }^{* *}$ IRCCyN: Nantes - France - www.irccyn.ec-nantes.fr
}

\begin{abstract}
Taking into account the variations of the environment is a means of improving performances of roll stabilisation systems. The ship behaviour is modelled as a MIMO LPV system. A methodology is presented which leads to a gain-scheduled control law. The synthesis is based on multi-objective optimisation, and on the representation of the standard system as a polytopical system, which depends on ship speed and on a stabilisation quality factor. Simulation results are given. Copyright @2004 IFAC
\end{abstract}

Keywords: Ship control; roll stabilisation; $\mathrm{H}_{\infty}$ control; Gain Scheduled Control; polytopic representation; LMI.

\section{INTRODUCTION}

A major improvement in the stabilisation systems' performances should be to adapt to the environmental conditions: waves (encounter angle, power, dominant frequency), ship speed, loading conditions. However, there is relatively few published documents on such control laws. Yet, the dependance on the ships speed has been described and used for many years (Lloyd, 1989; Grimble et al., 1993) for PID and $\mathrm{H}_{\infty}$ control laws. Manual mechanism to cope with changes in the sea state has been introduced (Blanke et al., 2000).

This document details the investigation realised for roll stabilisation towards the construction of a methodology to derived gain scheduling controllers. It is presented to be as general as possible with the aim to use direct informations about the environment. However, it will be applied on a frigate to obtain, in a first step, a controller with only two varying parameters, the ship speed and a stabilizing quality factor that could be tuned by any adaptation process taking into account the environment (manually or automatically). The context aims to be as realistic as possible, and is based on industrial data. The proposed methodology is based on $H_{\infty} / \mathrm{LMI}$ results (Apkarian and Gahinet, 1995). This is the sequel of the study proposed in (Tanguy et al., 2003), for varying conditions.
The paper is organized as follows: the process and its environment are shortly described in section 2 as a MIMO linear parameterically varying (LPV) system. The control methodology is detailed in section 3. A four steps methodology leads to the gain scheduled controller. It is applied in section 4 on a frigate type vessel. Simulation results and comparisons of performances with LTI $H_{\infty}$ controllers are described in section 5. Section 6 gives perspectives of improvement.

\section{MODEL}

A ship in a seaway can be modelled as a linear parameterically varying system. This section is a condensed description of the results of a chapter of (Tanguy, 2004).

Comprehensive models derived from hydrodynamics are too complex to be used in control. Thus, acceptable simplifying assumptions are made: amplitude of motions are small; the ship dynamics is independent of the swell frequency. Eventually, the roll motion is considered to be the superposition of the motions induced by the waves and the motions induced by the actuators. Perturbation motions will be taken as an additive disturbance on the outputs of the ship's dynamics, as shown on figure 1 . 


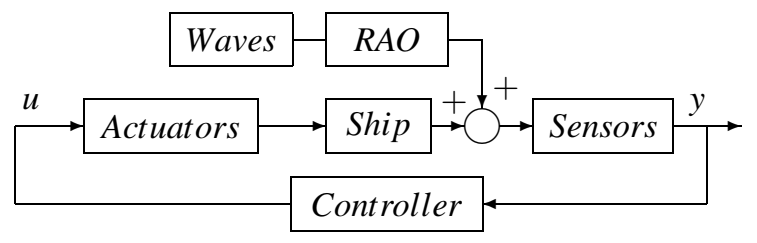

Figure 1. Control model with output disturbance.

\subsection{Sea disturbance}

Waves are the result of the sustained action of the wind over a wide sea surface. Complex sea states are considered to be the superposition of an infinite number of monochromatic waves. Waves will here be considered to be long crested.

Wave amplitude spectra, allow to characterise sea states all over the planet. The Bretschneider spectrum (Fossen, 1994), will be used in the simulations with parameters $H_{S}$ (wave height) and $\omega_{P}$ (the spectrum peak pulsation).

The wave spectrum encountered by a moving ship is different from the one seen by a motionless observer, due to Doppler effect. Parameters that modify it are: the ship speed $V$ and the encounter angle of waves $\psi_{e}$ :

$$
\omega_{e}=\omega\left(1-\omega \frac{V}{g} \cos \left(\psi_{e}\right)\right)
$$

\subsection{Synthesis and Simulation models}

2.2.1. Synthesis Model The synthesis model is linear with varying parameters. It is written as a state space model with state $x=[v, p, r, \phi, \psi, \alpha, \dot{\alpha}, \delta, \dot{\delta}]^{T}$ where $v, p, r$ are respectively the sway, roll and yaw velocity; $\phi$ and $\psi$ are the roll and yaw angles; $\alpha$ and $\delta$ are the actual position of the actuators (fins and rudders). The control variable, $u$, is the desired position of the actuators. The measures $y$ considered for control are the roll velocity $p$ and the angle $\psi$. The model is of the following type:

$$
\begin{aligned}
& \dot{x}=A(V) x+B u \\
& y=C x
\end{aligned}
$$

Only the coefficients of the matrice $A$ are dependent on $V$ as second (fins and rudders efficiency), first (damping) or zeroth (buoyancy) order polynomials. The synthesis model for the studied case of section 4 is only parameterised in speed $V$.

The coefficients are calculated from a seakeeping numerical code. Details on the construction and expressions of 2 and 3 are given in (Tanguy, 2004). Just note that the matrices are chosen for an encounter angle of $90 \mathrm{deg}$, that the load is constant. Moreover the dynamics of the actuators is modelled by a second order LTI system, and is integrated in the dynamics.
2.2.2. Simulation Model The simulation model is obtained by adding an output disturbance (motions due to waves) to $y$. Disturbance motions are computed using the Bretschneider spectrum with $T_{P}=10 \mathrm{~s}\left(\omega_{P}=\right.$ $\frac{2 \pi}{T_{P}}$ ) and $H_{s}=3.25 \mathrm{~m}$ (Sea State 5 in the northern Atlantic) and the motion RAO of the ship.

$$
y=\left(p+p_{w}, \psi+\psi_{w}\right)^{\top}
$$

In addition, the simulations takes into account the temporal non-linear aspects of saturation (in angle and rate for both the fins and rudders) and digitalisation of the control law. A pure delay is also added in temporal simulations to make up for the information transportation effects in the ship internal network.

\section{PROPOSED SYNTHESIS METHODOLOGY}

\subsection{Introduction}

The motions of the ship depends on its speed $(V)$, its direction $\left(\psi_{e}\right)$ and on the environment characterized by the sea state parameters $H_{S}$ and $T_{P}$. Gain scheduled controllers that depend on these parameters should be an interesting way to tackle the roll reduction. In general, controllers implemented are PIDs, tuned at the ship roll frequency (Lloyd, 1989; Katebi et $a l ., 2000)$. The dependance on ship speed is rarely described in the litterature (except for (Lloyd, 1989; Grimble et al., 1993; Blanke et al., 2000)). The gains generally are inversely proportionnal to the square of the ship speed. No theoretical proof of the closed loop stability exists in this case; but simulation tests "show in practice" the stability and the efficiency of the method.

In this study it is proposed to use the recent $H_{\infty} / \mathrm{LMI}$ techniques to compute gain scheduled controllers for Linear systems with varying parameters since they guarantee the closed loop stability. This section propose a methodology for the general considered problem. In the next section, its application to particular conditions will be detailed.

\subsection{The general specifications for the control law synthesis}

Specifications characterizing the desired behavior of the ship are chosen from mechanics and passengers' comfort matters:

- reduce the roll motion inside the roll bandwidth and do not amplify it outside,

- keep the yaw angle as constant as possible,

- do not use too much power,

- respect a given power repartition on the actuators. The fins are used only for roll stabilisation, and should interfere very little with the heading. On the contrary, rudders have a great influence 
on roll motions, but are primary used to control the yaw,

- tolerate only "acceptable" position and speed saturation of the actuators.

Others specifications are added from the control engineering point of view:

- the closed loop and the controller must be stable,

- some robustness properties are necessary against uncertainties (delay, discretisation...).

\subsection{A four step methodology}

In order to derive gain scheduled controllers from the now classical $H_{\infty} /$ LMI techniques, one needs a linear parameterically varying standard model defined from the dynamics of the ship (section 2) and weight functions, which have to be a translation of the previous specifications. Note that the main difficulty here is to give a proper translation of the physical specifications into mathematics. It is proposed to procede in two stages: first, compute the weights for fixed values of the varying parameters; then, compute the varying standard model with an interpolation technique.

More generally, the following four-stage methodology is proposed to achieve the final synthesis goal:

- Stage 1: Choose the varying parameters which will be considered. All the varying parameters appearing in the model (section 3.1) should be considered; but the more parameters, the more complicated will be the computation.

- Stage 2: Choose the parameters values in a grid. At each set of the parameters, determine the weights for the standard model that result in a $H_{\infty}$ controller such that specifications are fulfilled. This is based on the resolution of a multi-objective optimisation problem (Tanguy et al., 2003).

- Stage 3: Compute, with a classical interpolation technique, a linear standard model with varying parameters, from the fixed standard models resulting from stage 2 .

- Stage 4: Compute a gain scheduled controller for the linear varying parameters model with standard numerical code ${ }^{1}$.

Some technical aspects are now classical, and may be solved with standard existing Toolboxes: $H_{\infty}$ synthesis in stage 2 , and $H_{\infty} /$ LMI gain scheduled controller synthesis in stage 4 . The definition and the resolution of the multi-objective optimisation problem (in stage 2) resulting in optimised weights were already introduced in (Tanguy et al., 2003). The originality of the present paper lays in the whole methodology, which considers completely the variation of the parameters.

\footnotetext{
1 Matlab LMI toolbox, for example.
}

\section{APPLICATION OF THE METHODOLOGY}

The studied ship is a frigate (length $120 \mathrm{~m}$, displacement 3000 metric tons). The considered environmental conditions are sea state $5\left(T_{P}=10 \mathrm{~s}\right.$ and $H_{s}=$ $3.25 \mathrm{~m}$ ) for a encounter angle $\psi_{e}$ of $90 \mathrm{deg}$.

\subsection{The varying parameters}

Two parameters were used: the ship speed over the water, and a tuning parameter called Stabilisation Quality Factor (SQF). Dependance in the ship speed is needed. The SQF defines roll reduction quality: it is defined by the depth of the roll sensibility transfer function between an additive roll disturbance and the roll angle. Its value is intended to be directly tuned from the bridge or by an adaption process, taking into account power consumption, actuators' saturation levels, and sea state measurements.

The values of the parameters are defined by a comprehensive gridding, with steps every 5 knots in speed from 10 to 25 knots, and every 1 unit in SQF quality from 2 to 8 .

\subsection{Controller synthesis for fixed parameters}

A more comprehensive study (Tanguy et al., 2003), premise of the present article, gives the details for the computation of controllers for constant values of the parameters. The method used was to solve a multi-objective optimisation problem with an evolution strategy (genetic algorithm), and to choose a particular solution from strict guidelines. Another solution is used here (as the optimisation process may take quite some time to reach a reasonnably good value), though very close in principles to the former: the parameters' space is comprehensively studied, and gives good results in relatively short time.

\subsubsection{Definition of the multi-objective optimisation} problem The choice of the control law (here the weights of the $H_{\infty}$ standard problem) is defined as the result of a multi-objective optimisation problem under contraints. The objectives and contraints are derived from the specifications introduced in subsection 3.2:

O1 : Reduce the roll motion. It is expressed as the minimisation of the roll RMS value on a particular sea state for the closed loop system.

02: Use the minimum power. It is necessary to ensure that the two actuators do not compensate for one another, case which may appear (for MIMO PID, for instance). The sum of the RMS values of the fins' and rudders' positions (resp. $\sigma_{\alpha}$ and $\sigma_{\delta}$ ) is minimised, for the same sea-state.

O3 : Respect as precisely as possible the repartition constraint. The objective is defined as the weighted 
ratio 'use of the fins' over 'total use of the actuators', the weights being the $H_{\infty}$ norm of the open loop transfer functions between fin and rudder position and roll.

The constraints used, in addition to these objectives, are:

C1 : the controller must be stable;

C2 : the closed loop (system + controller), given a control application delay, must be stable;

C3 : the delay margin must be acceptable, in order to take into account digitalisation effects, and information transfer delay... The delay margin is evaluated with sensitivity and complementary sensitivity output transfer functions analysis, as the control problem is MIMO (see (Tanguy, 2004));

C4 : the amplification under and over the ship roll resonance must be low (Hearns et al., 2000); They are calculated from sensitivity transfer between $p_{w}$ and $p$;

C5 : the actuators may not endure too much saturation, in both position and velocity. It is not possible to determine exactly the saturations levels when working in the frequency domain, for it has only a temporal meaning. Yet, they can be evaluated from statistical considerations ((Lloyd, 1989; Price and Bishop, 1974)).

Note that this optimisation framework can be used to tune different type of controllers. It is here applied to tune $H_{\infty}$ controllers, but it has also been applied to MIMO PID.

4.2.2. Definition of the $H_{\infty}$ problem This part of the methodology is similar to the one detailed in (Tanguy, 2004), so it won't be precisely described here. Just note that the problem is set up as a mixed sensitivity problem (figure 2 ).

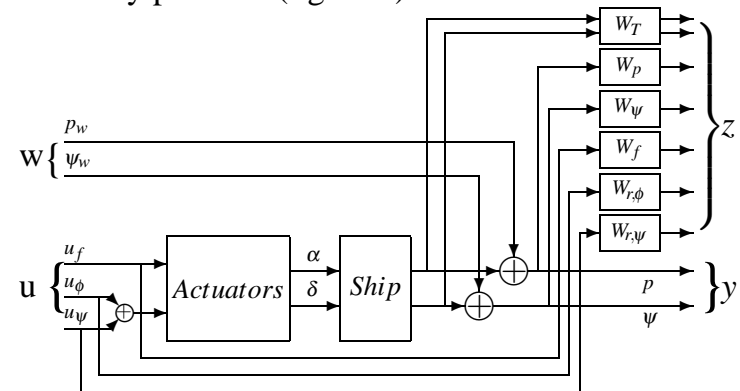

Figure 2. Mixed sensitivity problem.

Most of the parameters of the weights have a fixed value. In fact, only the gains of $W_{f}$ and $W_{r \phi}$ may vary $^{2}$ in the multi-objective optimisation problem. The shape of the roll derivative sensitivity weight $\left(W_{p}\right)$ has been carefully chosen(figure 3 ). The depth of the well is defined by the SQF which varies from 2 to 8 , its center by the roll resonance frequency of the ship; it is taken to be a constant; in a more complete study

2 This may appear very simple, yet this is the result of several tests!

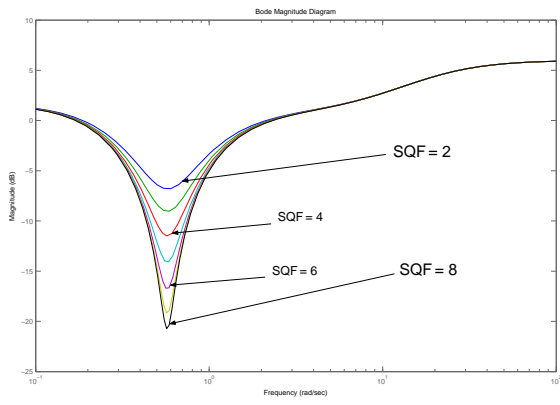

Figure 3. The roll derivative sensitivity $W_{p}$

it would be a fonction of the ship speed, the encounter angle of waves, the sea state...

4.2.3. Results The results of this step are not the $H_{\infty}$ controllers, even if they have to be calculated in order to assess their performances. The actual results are the weights themselves and the standard models, for every combination of parameters. They are the basis for the next step.

\subsection{Computation of the LPV model}

The computed standard models (dynamics augmented with frequency weights) show a dependency on $V$ and $V^{2}$ and $S Q F$. With a classical interpolation technique, it is possible to synthesise their expression in the following LPV model:

$$
\begin{aligned}
P\left(V, V^{2}, S Q F\right) & =\left[\begin{array}{ccc}
A\left(V, V^{2}, S Q F\right) & B_{1}\left(V, V^{2}, S Q F\right) & B_{2} \\
C_{1}\left(V, V^{2}, S Q F\right) & D_{11} & D_{12} \\
C_{2} & D_{21} & D_{22}
\end{array}\right] \\
& =P_{c}+P_{V} V+P_{V^{2}} V^{2}+P_{S Q F} S Q F
\end{aligned}
$$

A basic solution is to consider the model (5) as an affine model $P_{a}\left(X_{1}, X_{2}, X_{3}\right)$ obtained by replacing $V$ by $X_{1}, V^{2}$ by $X_{2}$ and $S Q F$ by $X_{3}$. The parameters $X_{1}, X_{2}$ and $X_{3}$ are supposed to be independent of each other ${ }^{3}$. This leads to a very conservative model.

A polytopic model has instead been used for which, there exists dedicated control law synthesis code ${ }^{4}$. The chosen model is put under the following form:

$$
P_{p}(\theta)=\left\{\sum_{i=1}^{7} \alpha_{i}(\theta) \pi_{i}, \alpha_{i}(\theta) \geq 0, \sum_{i=1}^{7} \alpha_{i}(\theta)=1\right\}
$$

where the vertices $\pi_{i}$ are the image of the vertices of a polytopic domain $\mathscr{P}$ which define acceptable restricted values of $\theta$ :

$\left\{\theta=\left(X_{1}, X_{2}, X_{3}\right) / \theta=\sum_{i=1}^{7} \alpha_{i} P_{i}, \alpha_{i} \geq 0, \sum_{i=1}^{7} \alpha_{i}=1\right\}$. The vertices $P_{i}$ of $\mathscr{P}$ have been chosen such that the possible values of $\left(V, V^{2}, S Q F\right)$ of the initial LPV model (5) are included in $\mathscr{P}$ (see figure 4). The facet $\left\{P_{4}, P_{5}, P_{7}\right\}$ express the constraint that for small values

\footnotetext{
3 With $X_{1} \in[1025], X_{2} \in[100625]$ and $X_{3} \in[28]$.
}

4 LMI toolbox of matlab, for example. 
of $V$ no good roll damping is possible ${ }^{5}$. The influence of $V^{2}$ remains sufficiently low to keep representation adequate (without too much conservatism).

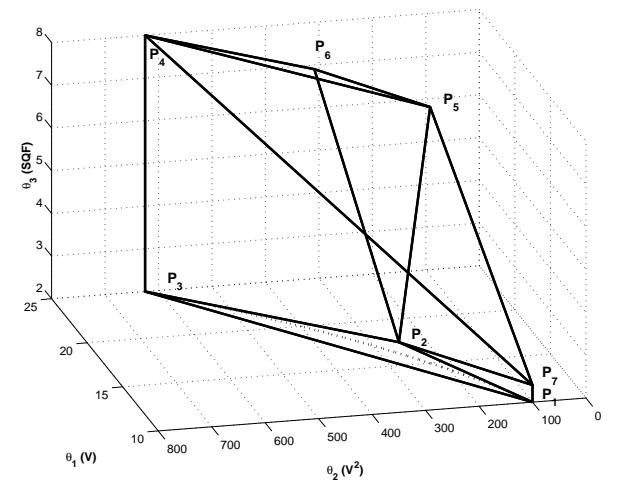

Figure 4. Polytope $\mathscr{P}$ (solid) and projection on $\left(\theta_{1}, \theta_{2}\right)$ (dashed).

\subsection{Computation of the gain scheduled controller}

Once a valid polytopic standard model is written, the computation of the polytopic controller is quite straightforward. The LMI Control toolbox of Matlab provides tools for such a work.

A quite similar method, and giving potentially better results, is to represent the LPV system with a LFT (Apkarian and Gahinet, 1995; Magni, 2001). The controller synthesis is clearly described in the litterature (and quite classical as for now), yet it requires a great quantity of computation and formalisation. Furthermore, controllers may exist without the theorem (and the equations) allowing to compute it.

\section{SIMULATION RESULTS AND COMPARISONS}

\subsection{Discrete form controller}

For the application of the controller on the simulation model, its matrices are computed at each time step. The discretisation is realised with a zero order hold approximation, which requires the computation of a matrix exponential: this method is surely not optimal, but works properly on a $800 \mathrm{MHz}$ computer, and it ensures stability of the controller.

\subsection{Simulations}

The temporal simulations are performed with $\psi_{e}=$ $90 \mathrm{deg}$ and sea state 5 (figures 5, 6, 7) or 6 (figure 8). Others simulations are presented in (Tanguy, 2004).

Figure 5 and 6 illustrate the behaviour of the Gain Scheduled Controller (GS) when the conditions vary.

\footnotetext{
5 This is the result of Stage 2.
}

Figure 5 presents the performances of the controller when the speed vary slowly from 10 to 20 knots and with a constant SQF (2). The activity of the actuators decrease with the speed. This physically corresponds to the fact that their efficiency is increasing with the speed (it is a function of $V^{2}$, see section 2.2.1), and that maximal fin deflection value decreases with speed (there exists a maximum allowed effort). This capability of the GS controller is not available with a LTIcontroller, see figure 7.

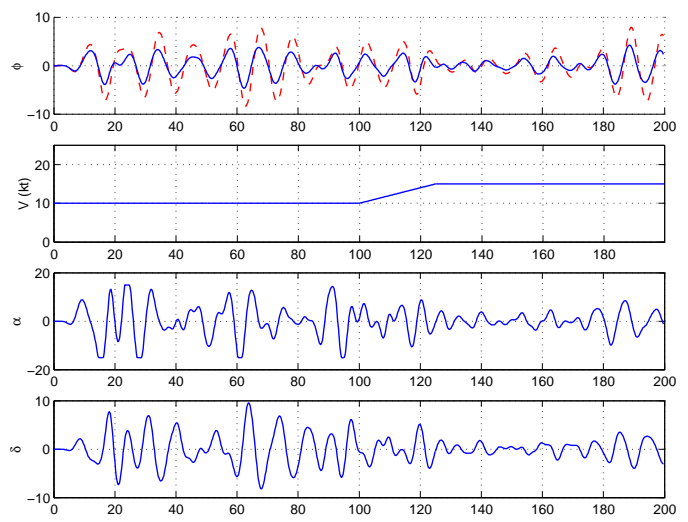

Figure 5. Variation of Speed during simulation. Closed loop (solid), open loop (dashed).

Figure 6 presents the effect of a variation of the SQF from 2 to 8 , the ship having a constant speed of 15 knots. Note that the variation can be infinitely fast without destabilising the loop, thanks to the LMI derivation of the controller. The effect of SQF is clear: better roll stabilisation is obtained from $t=150 \mathrm{~s}$, while maintaining acceptable increase of the actuators activity. In this case the increased activity was expected since the fin efficiency does not vary (constant speed).

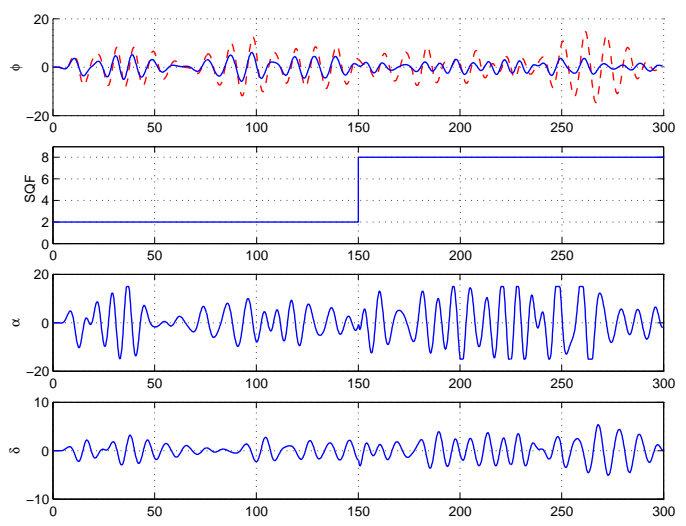

Figure 6. Variation of SQF during simulation. Closed loop (solid), open loop (dashed).

Figure 7 shows the interest of the gain scheduled controller when the ship speed vary from 15 knots to 25 knots, compared with a LTI- $H_{\infty}$ controller (tuned 
for a speed of 15 knots). The LTI- $H_{\infty}$ controller is optimised with the techniques of section 4.2 for a speed of $V=15 \mathrm{knots}$, for $\psi_{e}=90 \mathrm{deg}$ and for sea state 5. The SQF parameter of the GS controller is 8 . The roll attenuation does not change much with this controller, and as noticed previously the activity is a little bit decreased, because of the variation of fin efficiency. On the contrary, whereas the activity of the actuators were acceptable for the LTI- $H_{\infty}$ controller, for 15 knots, it becomes inacceptable for 25 knots: actuators are agitated (motions have a period of 3 seconds). The LTI- $H_{\infty}$ shows better roll reduction than the the GS controller, but the actuators saturate too often.

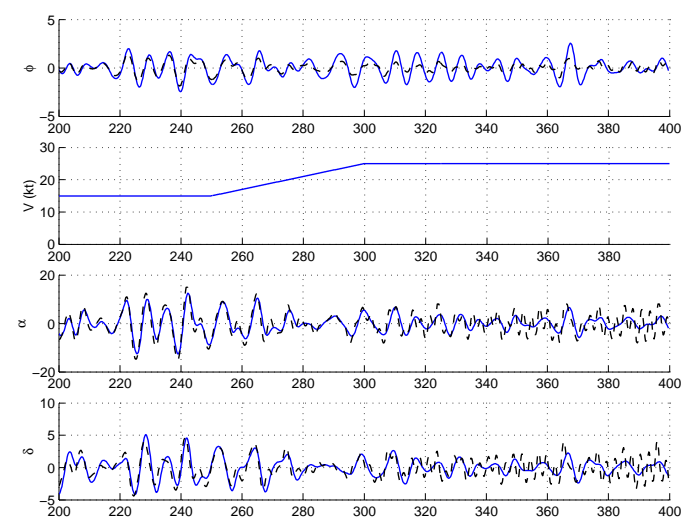

Figure 7. GS (solid) and LTI (dashed) controllers.

Moreover, figure 8 shows how an action on the SQF can be used to reduce the activity of the actuators. The two simulations shown on figure 8 correspond to the behaviour of the ship on a sea state 6 . The GS controller is adapted to sea state 5 . The solid curve corresponds to $S Q F=8$, and the dashed curve to $S Q F=2$. Downgrading the performances, in case of navigation on higher sea states than expected will show reduction of the saturation frequency and as a consequence will help protect of the actuators mechanics.
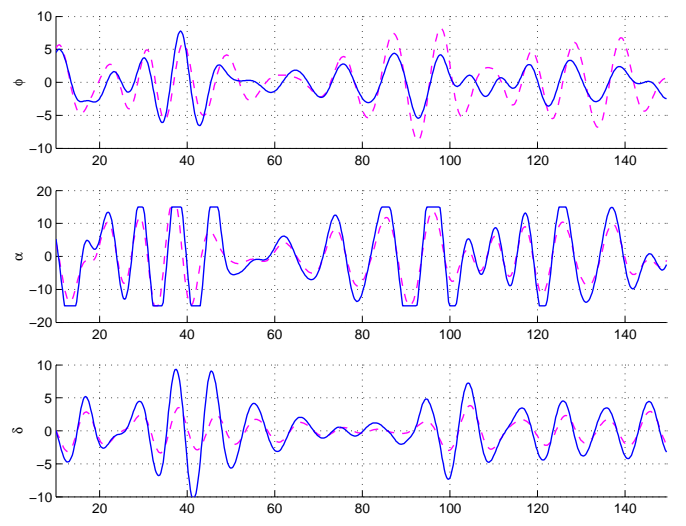

Figure 8. GS controller with different SQF. $S Q F=$ 2:dashed, 8:solid.

\section{CONCLUSION-PERSPECTIVES}

A methodology is given for computing a gain scheduled controller, based on LMI and on polytopic modelisation of the process. Parameters used are the ship speed and a stabilisation quality factor. The methodology has been applied to a frigate-like ship in simulation. Evolution of the parameters during the simulation is possible, which influence the controllers matrices, and thus the behaviour of the stabilised ship, allowing for example a good adaption to ship speed and reduction of saturation frequency. Improvement are expected from introducing more parameters (encounter angle, sea state...), in order to better monitor the behaviour of the control law. Another potential way of improvement is the use of LFT representation for modelling and control law synthesis.

\section{REFERENCES}

Apkarian, P. and P. Gahinet (1995). A convex characterization of gain-scheduled $h_{\infty}$ controllers. IEEE TAC 40(5), 853-864.

Blanke, M., J. Adrian, K.E. Larsen and J. Bentsen (2000). Rudder roll damping in coastal region sea conditions. In: Proceedings of MCMC 2000. Aalgorg, Danemark.

Fossen, T. I. (1994). Navigation and Guidance of Ocean Vehicles. John Wiley \& sons. New York.

Grimble, M.J., M.R. Katebi and Y. Zhang (1993). $h_{\infty}$ based ship fin-rudder roll stabilisation design. In: 10th Ship Control Systems Symposium. Vol. 5. Ottawa, Canada.

Hearns, G., R. Katebi and M.J. Grimble (2000). Robust fin roll stabiliser controller design. In: 5 th IFAC Conference on Manoeuvering and Control of Marine Crafts. Aalborg, Danmark.

Katebi, M.R., N.A. Hickey and M.J. Grimble (2000). Evaluation of fin roll stabiliser controller design. In: 5th IFAC Conference on Manoeuvering and Control of Marine Crafts. Aalborg, Danmark.

Lloyd, A.R.J.M. (1989). Seakeeping, Ship Behaviour in Rough Weather. Marine Technology. Hellis Horwood.

Magni, J.F. (2001). Linear Fractional Representations with a Toolbox for Use with MATLAB. Toulouse, France. Technical report TR 240/2001 DCSD.

Price, W.G. and R.E.D. Bishop (1974). Probabilistic Theory of Ship Dynamics. Chapman and Hall. Londres.

Tanguy, H. (2004). synthèse de lois de commande à gains programmés pour la stabilisation en roulis des navires. PhD thesis. Université de Nantes, École Centrale de Nantes. Nantes, France.

Tanguy, H., G. Lebret and O. Doucy (2003). Multiobjective optimisation of pid and $h_{\infty}$ fin/rudder roll controller. In: Proceedings of MCMC 2003. Girona, Spain. 